\title{
The Strategy of Solving Smaller, Similar Problems in the Context of Combinatorial Enumeration
}

\author{
Elise Lockwood ${ }^{1}$
}

Published online: 29 September 2015

(C) Springer International Publishing Switzerland 2015

\begin{abstract}
Combinatorial enumeration has a variety of important applications, but there is much evidence indicating that students struggle with solving counting problems. The roots of such difficulty, as well as ways to mitigate such difficulty, have not yet been thoroughly studied. In this paper, one particular aspect of students' counting activity is explored - the use of the problem-solving heuristic of solving smaller, similar problems. Drawing upon student data from clinical interviews in which post-secondary students solved counting problems, four ways in which students successfully utilized this strategy are described. Additionally, three potential pitfalls of the strategy are identified and discussed. Finally, the case is made that the strategy is particularly useful within the area of combinatorics.
\end{abstract}

Keywords Combinatorics $\cdot$ Counting problems $\cdot$ Problem solving Discrete mathematics - Smaller cases

\section{Introduction and Motivation}

Combinatorial topics have relevant applications in areas such as computer science and probability (e.g., Jones 2005; Polaki 2005), and they provide worthwhile contexts in which students can engage in meaningful problem solving. As such, combinatorics has received attention in both $\mathrm{K}-12$ and undergraduate curricula, emerging as an important aspect of a well-rounded curriculum (e.g., National Governors Association Center for Best Practices and Council of Chief State School Officers 2010; NCTM 1989; 2000). Research indicates, however, that students face difficulties with combinatorial concepts, and this is certainly true at the undergraduate level. Godino et al. (2005) note that in

Elise Lockwood

elise314@gmail.com

1 Department of Mathematics, Oregon State University, Corvallis, OR, USA 
Roa's (2000) study, 118 undergraduate mathematics majors were given a questionnaire with 13 "simple" combinatorial problems (Godino et al. 2005, p. 4). They note that these students "generally found it difficult to solve the problems (each student only solved an average number of 6 problems correctly)" (p. 4). In their study on undergraduates, Eizenberg and Zaslavsky's findings similarly "support the assertion that combinatorics is a complex topic - only 43 of the 108 initial solutions were correct" (2004, p. 31). Additionally, Hadar and Hadass (1981) state that, "combinatorics is a field which most of the students find very complicated" (p. 436). Other researchers (e.g., Eizenberg and Zaslavsky 2004; Lockwood and Gibson 2014; Hadar and Hadass 1981; Kavousian 2008) concur with the claim that combinatorial problems are nontrivial for students, and some (e.g., Batanero et al. 1997) propose potential reasons for such difficulties. Given the fact that discrete and combinatorial topics are something with which mathematics and computer science majors should have facility, the reported success rates are alarming.

Beyond the potential role of combinatorics to help students succeed as computer programmers or statistically literate citizens, there is something about the domain of combinatorics that fosters deep and profound reasoning. Indeed, Sriraram and English (2004) note that, "Combinatorial problems ... constitute a rich opportunity for mathematical exploration" (p. 189). In his undergraduate textbook Applied Combinatorics, Tucker (2002) says of his counting chapter, "We discuss counting problems for which no specific theory exists" (p. 169). He further notes that "facility with these three basic skills in problem solving [logical reasoning, clever insights, and mathematical modeling], as much as an inventory of special techniques, is the key to success in most combinatorial applications." Finally, Tucker calls the counting chapter "the most challenging and most valuable chapter in this book" (p. 169). Combinatorics as a discipline is an ideal setting for fostering meaningful problem solving and rich mathematical thinking. As such, research on the teaching and learning of combinatorics warrants further attention in the mathematics education community.

There is much to learn about students' combinatorial thinking and activity. Researchers interested in the teaching and learning of combinatorics must not merely look for quick fixes to improve students' performances, but rather they should seek ways in which to strengthen students' combinatorial thinking and activity, both to deepen students' conceptual understanding and to equip them with meaningful approaches with which to solve combinatorial problems. There is a genuine need for researchers to look more closely at students' work on combinatorial problems - to identify specific areas of struggle for students and to attend to potential ways in which students may improve.

In this paper, I narrow the focus to one particular factor that is prevalent in students' solving of combinatorial problems - the use of the problem solving heuristic of solving smaller, similar problems. The research goal addressed in this paper is to identify and describe a variety of ways in which students utilize the problem solving strategy of

\footnotetext{
${ }^{1}$ Godino et al. (2005) do not explain what they mean by simple. It appears to mean that the problems were straightforward, focusing on a particular combinatorial operation (such as combination, permutation, etc.), free of any particularly tricky or deceptive elements. An example of such a problem is found on page 9: "Problem 3: A boy has four different colored cars (black, orange, white and green) and he decides to give out the cars to his friends Fernando, Luis and Teresa. In how many different ways can he distribute the cars? For example, he could give all the cars to Luis."
} 
working through a smaller problem while solving combinatorial tasks. I seek to contribute both to the combinatorics education literature and to the problem solving literature - to shed light on the problem solving strategy of using smaller problems, and to provide particular insight into students' combinatorial activity. Even more, I make the case that work with smaller problems serves as an especially significant problem solving strategy within the domain of combinatorial enumeration.

\section{Literature Review and Theoretical Perspective}

By a strategy of solving smaller, similar problems, I mean the problem solving technique of attempting one or more simpler versions of a problem as a means of gaining insight into a solution technique that may apply to the original problem. To further clarify what is meant by "smaller, similar problems," consider the following. In any given counting problem, there are typically a number of certain conditions that specify what the problem is asking. These conditions refer to the rules or limitations that must be met in a given problem. Some of these might be numerical in nature (e.g., the specific number of letters in a password, or number of choices of shirts or pants for an outfit), but others might refer to non-numerical conditions (e.g., the fact that repetition of letters is allowed in a password). For clarity, I refer to numerical conditions as parameters and to non-numerical conditions as constraints. In relation to a given problem, any other problem that a student might attempt to solve will be called "smaller" if it reduces one or more of the parameters in some way, and will be called "similar" if it generally maintains the constraints of the original problem. It is noteworthy that the numerical reduction of the parameters may indeed affect certain mathematical properties of those conditions, sometimes in unexpected ways. For instance, if a student reduced a composite number (like 100) to a smaller prime number (like 3), there may be some unexpected properties about the small prime number that would not carry over to the original problem.

To engage in the small cases strategy discussed in this paper, I mean that a student will, as an intentional strategy, implement the following steps: a) identify a smaller problem that numerically reduces one or more of the parameters of the problem but maintains the overall constraints of the original problem, b) attempt to solve that smaller problem, and c) apply what he or she learned in solving that smaller problem to the original problem. Put less technically, the smaller problem should involve smaller numbers, but it should maintain the overall rules or purpose (one could say the gist) of the original problem. At times I also refer to this as "solving a smaller case" because it represents solving a smaller instance or case of the original problem. ${ }^{2}$

As an example of the smaller, similar problem strategy studied here, an original problem may be, "How many 6-character license plates can be made, where each character can be any capital letter or any numerical digit? Repetition of characters is allowed." The primary conditions of the problem are that there are a certain number of characters (six), there are a certain number of options for each character $(36 ; 26$ capital

\footnotetext{
${ }^{2}$ Observe that another strategy may involve simplifying the constraints while maintaining the parameters, or performing a combination of these modifications. This strategy is fundamentally different in many ways, however, and so will not be discussed in this paper.
} 
letters and 10 numerical digits), repetition of those characters is allowed, and, because they are license plates, the order of non-identical characters matters (ABCDEF would be a different license plate from FEDCBA). A student might reduce the original problem to "How many 4-character license plates can be made, where each character must be $\mathrm{A}, \mathrm{B}$, or $\mathrm{C}$, where repetition is allowed?" Here, the student numerically reduces two of the parameters (the number of characters and the number of choices for those characters), and yet maintains the overall constraints of the problems (that repetition is not allowed and that license plates are the objects being counted).

When talking about reducing problems to smaller, similar problems, an important issue is whether the similarity exists in the eyes of the student or the researcher. I have previously suggested (Lockwood 2011b) that actor-oriented transfer (AOT) is a valuable lens through which to examine students' combinatorial problem solving. Lobato (2003) introduced the notion of AOT, describing a shift from "an observer's (expert's) viewpoint to an actor's (learner's) viewpoint by seeking to understand the processes by which individuals generate their own similarities between problems" ( $p$. 18 , emphasis in original). In this view, the researcher focuses on student-generated connections between problems, and not on connections that the researcher may expect. Subsequently in this paper, as I discuss connections that students make between original problems and smaller problems, I atte mpt to look at such connections from the students' perspectives. That is, drawing upon Lobato's $(2003,2012)$ work with actor-oriented transfer, in this study I seek to judge student-generated similarities among problems, not relationships that I myself determine to be similar.

Silver $(1979 ; 1981)$ examined the solving of "related" problems by having middle school students categorize sets of 16 to 24 algebra word problems. He found direct relationships between students' successes in problem solving and ability to categorize problems according to mathematical structure; in these studies, the problems were judged as related by experts, not by the students themselves. The specific strategy of solving smaller related problems has been alluded to by problem solving researchers like Schoenfeld $(1979,1980)$ and Polya (1945). Polya discusses this strategy in terms of "discovering a simpler analogous problem" (p. 38, emphasis in original) and suggests that solving such a problem provides a model to follow when solving the original problem. Schoenfeld (1980) also lists as frequently used problem solving heuristics both considering "slightly modified" and "broadly modified" problems (p. 801). Additionally, Schoenfeld (1979) conducted a study examining the effectiveness of explicitly teaching problem solving heuristics, and considering "a smaller problem with fewer variables" (p. 178) was one such heuristic that he examined. Schoenfeld's and Polya's attention to such a strategy suggests that it could be valuable for problem solving across a variety of mathematical domains.

In the combinatorics literature, little has been investigated about the use of smaller problems in the domain of counting problems. While no studies have explicitly looked at the use of small cases in the context of solving such problems, there is one study that alludes to such a strategy. Eizenberg and Zaslavsky (2004) examined undergraduate students' verification strategies on combinatorial tasks. One of those strategies they identified was "Verification by modeling some components of the solution" (p. 26), and one aspect of this type of verification involved applying "the same solution method by using smaller numbers" (p. 26). Eizenberg and Zaslavsky provide an example of an expert mathematician effectively using this strategy, but they are quick to point out that 
keeping the structural integrity of a problem when going to smaller numbers can be tricky - particularly with combinatorial problems. Indeed, they note that while the strategy "could be very powerful...it requires deep structural consideration." They go on to say that "We speculate that although it may seem natural to students to employ this strategy (as indeed some tried to), applying it correctly needs direct and systematic learning" (p. 32).

In their longitudinal study, Maher and her colleagues (e.g., Maher and Martino 1996; Maher et al. 2011) have emphasized ways in which carefully chosen tasks can foster reasoning and proof among even very young students. Their work has seen students generalize using smaller problems, and it suggests that meaningful representations and structures can form key mathematical relationships for students. They show, for instance, that students referred to prototypical problem types even years after their initial work on that problem (e.g., students refer to "the towers problem" in Maher and Speiser 2002). However, these researchers have not yet isolated the particular strategy of solving smaller, similar problems as a relevant factor of student work with particular domain-specific implications.

Additionally, combinatorially, I frame this paper within my notion of a set-oriented perspective (as discussed in Lockwood 2013; 2014). I advocate a perspective that the activity of counting can be viewed as inherently involving the enumeration of sets of outcomes (see also Hadar and Hadass 1981; Polaki 2005), and that solving counting problems can be framed in light of organizing and structuring those outcomes. I provide evidence that there is much benefit for students in considering sets of outcomes as they count. Drawing upon this work, then, I relate the findings below to the perspective that activities related to sets of outcomes (such as systematically listing) can be beneficial for students' success on counting problems. The studies discussed above not only situate my work, but they also serve as a theoretical framework, guiding the analysis of the data and the discussion of the results.

\section{Methods}

\section{Participants}

In the study described in this paper, I interviewed 22 post-secondary students (12 undergraduates and 10 graduate students) as they solved combinatorial tasks. These students were recruited from graduate and undergraduate mathematics courses at a large, public university in the western United States. They were chosen based on their willingness to participate and on their familiarity with basic combinatorial concepts such as binomial coefficients (as determined by a recruitment survey). All of them had taken at least one discrete mathematics course, and some had taken courses in combinatorics or graph theory. Two undergraduates were computer science majors, the rest of the participants studied mathematics. I had initially interviewed eight undergraduate students, and the tasks proved to be challenging for them. In attempting to collect rich data and to achieve theoretical saturation (Auerbach and Silverstein 2003), I extended my recruiting to graduate students as well.

Each of these students participated in individual, videotaped, 60-90 min-long semistructured interviews. Fontana and Frey (2005) differentiate between structured 
interviews, in which the interviewer "asks all respondents the same series of preestablished questions with a limited set of response categories" (p. 701-2), and unstructured interviews, which are "open-ended, in-depth (ethnographic) interview[s]" (p. 705). Semi-structured interviews typically involve "an interview guide as opposed to a fully scripted questionnaire" (Willis 2005, p. 20), and I chose such interviews to allow for flexibility that could allow me to adapt to students' responses. In my study, the structure of the interviews was first to give students five combinatorial problems to solve on their own, and they solved all of these problems in succession in the first half of the interview. During this time, I encouraged the students to think aloud as they worked, and at times I asked clarifying questions or asked them to explain their work. My questions during the interviews were not intended to explicitly teach or instruct, but rather to elicit students' thinking.

After they had completed work on the five problems, the students subsequently returned to a subset of these problems, during which time I presented alternative answers for the students to evaluate. I chose which problems we revisited, and this decision was made in the moment, based on a student's work in a given interview. Factors that affected this choice of which problem to revisit included: students' initial answers (for instance, if a student arrived at one of the anticipated correct (or incorrect) answers, I might present them with an incorrect (or correct) answer); my perceived trajectory of a student's work on a given problem (for instance, if a student had been incorrect on a problem but had displayed some promising line of reasoning, I might ask them to revisit that task); and my perceived notion for what tasks would yield the richest data (for instance, if a student had been particularly detailed or verbose in their explanation of a certain problem, I might have asked them to revisit that problem). My content knowledge of the material enabled me to make informed decisions about which tasks to pursue.

Typically, having students revisit problems resulted in students comparing two answers, both of which could seem reasonable, but one of which contained an error. The motivation for this design was based on a desire to put students in a situation in which they had to evaluate incorrect but seemingly reasonable answers. Furthermore, the design facilitated ample opportunity for students to consider the problems and to express their thinking. Further details of the study can be found in Lockwood (2011a).

\section{Tasks}

While students in the study were given five tasks, here I focus on only two of the tasks (the Passwords problem and the Groups of Students problem); all of the following data excerpts refer to one of these problems. Below, for each problem, I briefly provide both a correct answer and an incorrect answer that are most relevant to subsequent discussion, acknowledging that there are in fact many correct and incorrect answers that could be presented. Both of these problems were adapted from upper-undergraduate or graduate level texts (Martin 2001; Tucker 2002) and require some knowledge of combinatorial concepts to be solved efficiently.

The Passwords Problem The Passwords problem states, "A password consists of 8 upper-case letters. How many such 8-letter passwords contain at least three Es?" The at least constraint is a noteworthy one. Because of this constraint, one solution is to break 
the problem into cases, ${ }^{3}$ in which the passwords contain three, four, five, six, seven, or eight Es. For any of those cases, the number of passwords containing $k$ Es is found by choosing spots for those Es to go (there are $\left(\begin{array}{l}8 \\ k\end{array}\right)$ ways to do this), and then filling in the remaining 8- $k$ spots with any of the 25 letters that are not E. Therefore, a correct result (subsequently referred to as Expression PC for Passwords Correct) is

$$
\sum_{k=3}^{8}\left(\begin{array}{l}
8 \\
k
\end{array}\right) 25^{8-k}=\left(\begin{array}{l}
8 \\
3
\end{array}\right) \cdot 25^{5}+\left(\begin{array}{l}
8 \\
4
\end{array}\right) \cdot 25^{4}+\left(\begin{array}{l}
8 \\
5
\end{array}\right) \cdot 25^{3}+\left(\begin{array}{l}
8 \\
6
\end{array}\right) \cdot 25^{2}+\left(\begin{array}{l}
8 \\
7
\end{array}\right) \cdot 25^{1}+\left(\begin{array}{l}
8 \\
8
\end{array}\right) \cdot 25^{0} .
$$

There is also a tempting solution that does not involve a case breakdown, which reflects a subtle error. Namely, the answer $\left(\begin{array}{l}8 \\ 3\end{array}\right) \cdot 26^{5}$ (subsequently referred to as Expression PI for Passwords Incorrect) can be argued by first choosing where to put three Es (there are $\left(\begin{array}{l}8 \\ 3\end{array}\right)$ ways to do this), there are guaranteed to be at least three Es in the password. Therefore, the remaining five letters could be any letter, including an $\mathrm{E}$ (hence, ). However, the problem with this answer is that some particular solutions get counted more than once. For example, the solution $\underline{E} \underline{E} \underline{E} \underline{A} \underline{B} \underline{E} \underline{E} \underline{E}$ gets counted multiple times, both when the first three Es were chosen $\left(\underline{E} \underline{E}-{ }_{-}-{ }_{-}\right)$and the rest of the word was filled in with ABEEE, and then again when the last three Es were chosen $\left(L_{-}-\underline{E} \underline{E} \underline{E}\right)$ and the rest of the word was filled in with EEEAB. To get a sense of the magnitude of these expressions, Expression PC yields 575,111,451, while Expression PI gives 665,357,056.

The Groups of Students Problem 4 The Groups of Students problem states, "In how many ways can you split a class of 20 into 4 groups of 5?" A correct answer to this problem (subsequently referred to as Expression GC for Groups Correct) is

$$
\frac{\left(\begin{array}{c}
20 \\
5
\end{array}\right) \cdot\left(\begin{array}{c}
15 \\
5
\end{array}\right) \cdot\left(\begin{array}{c}
10 \\
5
\end{array}\right) \cdot\left(\begin{array}{l}
5 \\
5
\end{array}\right)}{4 !} .
$$

To arrive at this solution, five students can be chosen to be in a group, there are $\left(\begin{array}{c}20 \\ 5\end{array}\right)$ ways to do this, then 5 of the remaining students are chosen to be in another group, $\left(\begin{array}{c}15 \\ 5\end{array}\right)$, then five more to be in a group, $\left(\begin{array}{c}10 \\ 5\end{array}\right)$, and then finally the last five to be in a group, $\left(\begin{array}{l}5 \\ 5\end{array}\right)$. However, the product must be divided by 4 factorial because the

\footnotetext{
${ }^{3}$ In the discussion of this problem, the word "case" indicates a strategy of breaking the original problem into disjoint sub-problems, which together comprise the entire problem; a "case" in this context suggests one of these sub-problems. This is a separate use of the word "case" than the remainder of the paper, in which a smaller "case" is meant as a smaller instance of the larger problem.

${ }^{4}$ A more detailed discussion of set partition problems such as the Groups of Students problem, see Lockwood and Caughman 2015.
} 
groups are not meant to be labeled or distinguished in any way - there is not a Group 1, Group 2, Group 3, and Group 4.

In order to see the need to divide by 4 factorial, particular outcomes can be considered. Suppose the first group that was chosen consisted of kids A, B, C, D, E, (call it Group A), the second group, kids F, G, H, I, J (Group F), the third group, kids K, L, M, N, O (Group K), and the fourth group, kids P, Q, R, S, T (Group P). Then this solution could be written as AFK, and it denotes the order in which the groups were chosen. However, suppose that each of the groups were the same (Group A still consists of A, B, C, D, E, etc.), but that they were chosen in a different order. That is, suppose the solution instead was KAFP or PAKF, representing the same groups that were picked in different orders. The problem only asks for groups of children; there is no first group, second group, etc. Therefore, if the particular division of the class is AFKP, it is the same division as KAFP. In fact, such a division occurs exactly 4 factorial ways (the number of ways to arrange the sequence of letters A, F, K, P). Division by 4 factorial thus ensures that each solution gets counted once, as it should be.

A typical incorrect solution (subsequently referred to as Expression GI for Groups Incorrect) is

$$
\left(\begin{array}{c}
20 \\
5
\end{array}\right) \cdot\left(\begin{array}{c}
15 \\
5
\end{array}\right) \cdot\left(\begin{array}{c}
10 \\
5
\end{array}\right) \cdot\left(\begin{array}{l}
5 \\
5
\end{array}\right),
$$

where the division by 4 factorial is neglected. The subtlety of the discussion above highlights the fact that this mistake could easily occur, and this possibility for error made it a particularly desirable problem for the study. Again, to get a sense of the size of these numbers, Expression GC gives 488,864,376 and Expression GI yields $11,732,745,024$.

\section{Data Analysis}

Initial data analysis involved transcription of the videotape excerpts. Then, in line with the methodological framework of grounded theory (Auerbach and Silverstein 2003; Strauss and Corbin 1998), I examined the data carefully and looked for phenomena that might be organized into themes. As this was a part of a larger study (other findings are reported elsewhere in Lockwood 2011a), I had initially gone through the entire set of transcripts and videotapes, coding noteworthy recurring ideas. The notion of small cases presented in this study was one such emergent theme, and I flagged episodes that represented instances of students' work with smaller cases. For the writing of this paper, I focused on these episodes, re-reading the transcripts and further familiarizing myself with instances in which students used small cases. I then re-watched the videos of these relevant excerpts, reviewed the transcripts, and categorized pertinent phenomena that I observed through this process. What emerged were particularly relevant instances of student work that highlighted various aspects of students' uses of small cases in their work on advanced combinatorial problems.

Grounded theorists Auerbach and Silverstein (2003) refer to abstract groups of themes as theoretical constructs. These theoretical constructs can then be organized 
into a theoretical narrative, "which summarize(s) what we have learned about our research concerns" (p. 40). That is, the narrative, which is based on theoretical constructs, can give a sense of the connections between the raw data and research questions. Following their methodological recommendations, I organized phenomena into themes and ultimately constructed a narrative about the role and relevance of small cases for students in the study.

\section{Results and Discussion}

I organize this discussion section into three major parts. First, I briefly provide general findings on the frequency and the extent to which small cases arose in the data. Second, I discuss features and benefits of small cases within the domain of combinatorial problem solving, drawing upon examples from the data to do so (specifically, students' work on the Passwords and Groups of Students problems). Third, I discuss potential dangers of using smaller cases in an imprecise manner, again illustrating with examples from the data.

\section{General Findings}

The data consist of 103 instances of students working on a counting problem (22 students working through five problems each, with some exceptions due to time constraints). The overall success rate was not high - only 42 of 103 initial solutions were correct. Of all of the 103 instances, there were 15 total times in which students referred to small cases. In 14 of these occurrences, the student identified and attempted to solve a smaller, similar problem, and then applied what he or she learned in solving that smaller problem to the original problem. In one instance a student used smaller, similar problems but did not relate his work back to the original problem. Table 1 below shows the students who used the strategy, which problems they worked on as they used the strategy, whether the problem properly reduced numerical parameters while maintaining constraints, and whether they related the problem to the original problem. In this table, I am counting at most one instance per student per problem - there is a 1 in a cell if the student used smaller cases on a given problem in any capacity. ${ }^{5}$

It is also noteworthy that the strategy was relatively infrequently used. While for some students this seemed to be a strategy that they regularly employed (some students said as much), many of the students did not seem to use it at all. That is, although small cases arose a total of 15 times, only ten (six graduate and four undergraduate students) of the 22 students drew upon the strategy, meaning 12 students never used small cases in their interviews. However, in spite of the fact that the strategy seems to have been

\footnotetext{
5 The reader may note that with the exception of Owen's work on the Cards problem, all of the instances of small cases arose in the context of either the Groups of Students problem or the Passwords problem. While students solved three other problems in the interviews, the small cases strategy did not arise in those contexts. I suspect that this is due largely to the fact that the other problems involved numbers that were relatively small in magnitude. The correct answers to the other problems were 153, 226, and 53, compared to 575,111,451 for the Passwords problem and 488,864,376 for the Groups of Students problem.
} 
Table 1 Students who used the strategy of solving smaller problems

\begin{tabular}{|c|c|c|c|}
\hline $\begin{array}{l}\text { Student (Undergraduate } \\
\text { or Graduate) }\end{array}$ & Problem & $\begin{array}{l}\text { Worked on a smaller, } \\
\text { similar problem }\end{array}$ & $\begin{array}{l}\text { Related the problem } \\
\text { to the original problem }\end{array}$ \\
\hline Aiden (U) & Groups of Students & 1 & 1 \\
\hline \multirow[t]{2}{*}{ Anderson (U) } & Groups of Students & 1 & 1 \\
\hline & Passwords & 1 & 1 \\
\hline Jenny $(\mathrm{G})$ & Passwords & 1 & 1 \\
\hline \multirow[t]{2}{*}{ Keith (U) } & Groups of Students & 1 & 1 \\
\hline & Passwords & 1 & 1 \\
\hline Matthew (G) & Groups of Students & 1 & 1 \\
\hline $\operatorname{Mia}(G)$ & Groups of Students & 1 & 1 \\
\hline Mike (U) & Groups of Students & 1 & 1 \\
\hline \multirow[t]{2}{*}{ Owen (G) } & Cards Passwords & 1 & 0 \\
\hline & Groups of Students & 1 & 1 \\
\hline \multirow[t]{2}{*}{ Paige (G) } & Groups of Students & 1 & 1 \\
\hline & Passwords & 1 & 1 \\
\hline \multirow[t]{3}{*}{ Zach (G) } & Groups of Students & 1 & 1 \\
\hline & Passwords & 1 & 1 \\
\hline & TOTALS & 15 & 14 \\
\hline
\end{tabular}

underdeveloped and underutilized among the students in my study, the strategy overwhelmingly helped the students who chose to implement it. Given students' overall difficulties on the problems, then, and given the fact that the use of smaller cases seemed to help some students, I contend that the strategy is worth examining as a potentially powerful aspect of combinatorial problem solving.

\section{Ways in Which Students Use Small Cases}

In this section I discuss ways in which student utilized small cases as they solved combinatorial problems. The examples below highlight some of the benefits of using small cases. These include using small cases: a) to facilitate systematic listing (which can be used for detecting useful patterns or identifying an overcount), b) to tackle one particular aspect of the problem, c) to explain the difference between two discrepant answers, and d) as a means of articulation, communication, and explanation.

Using Small Cases to Facilitate Systematic Listing Systematic listing involves the listing of outcomes in an organized way. There were a handful of instances in which students' uses of small cases facilitated systematic listing, and this activity clearly benefitted them in their solving of a given problem. This systematic listing helped students in two particular ways: a) to detect useful patterns, and b) to address an error of overcounting. I provide examples of each of these types below.

Systematic Listing (in Smaller Cases) as a Means to Detect Useful Patterns in Generating a Solution One way in which students utilized smaller cases was to detect 
useful patterns when generating a solution. ${ }^{6}$ Below we see Paige's reflection on the use of a smaller case as she discusses pattern formulation.

P: In counting problems this is a regular occurrence, where I will break it down into something small, try and figure out the pattern on a smaller level, and then apply that pattern up.

As a specific example of this method, in Anderson's work on the Groups of Students problem, he spent considerable time and energy listing out particular outcomes in the context of smaller cases. Having initially been overwhelmed by the original problem, Anderson ended up methodically building from smaller to larger cases, hoping to detect a pattern in the numbers that he could generalize. Specifically, Anderson began with considering having four people in the class, split into four groups, and he reasoned that there is one way to do that. He then bumped the problem up to eight people split into four groups, and began to list out some of the ways to split up eight letters (A through $\mathrm{H}$ ) into four groups. As we see underlined below, however, the 8-person case was too large for him to manage.

A: So I guess let's bump it up to 8. So now, if I take the first 8 letters of the alphabet, we can have AB CD EF and GH. Sure that's fine. We'd have AB CD EG and FH. Sure, and then we have AB CD EH FG. See this is, this is where is starts boggling me, it's because when we take out the separate groups and we're trying to count how many we can have.

To scale back some, Anderson decided to decrease the number of groups so as to make the problem more tractable. He started with dividing a class of four into two groups, and through listing found that there were three ways to do this. He then increased the problem to a class of six being split into two groups, and through careful systematic listing he found that there are 10 such possibilities. Anderson continued in this way, and at the heart of this work was pattern recognition - he was searching for a pattern in the numbers in order to generate the answer. While we ultimately ran short of time in the interview before Anderson could entirely finish this problem, I judge that he was on a very productive path toward making meaningful progress on the problem. Indeed, he said, 'I just haven't figured it out yet. I'd need to play with more examples to see if I can find something that lines up".

It is noteworthy, too, that when we revisited the problem, and I presented Anderson with the alternative answers to the problem, he related them to the work and patterns he had generated initially. When it came to making sense of the solutions, then, it seemed as though his involved work of systematic listing and looking for patterns was instrumental in helping him to understand the problem generally. The experimentation ultimately led to better understanding of the problem for Anderson.

Systematic Listing (in Smaller Cases) as a Means to Identify an Overcount In solving the Groups of Students problem, Mia had initially arrived at Expression GI, and

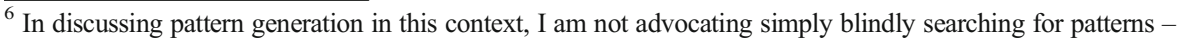
manipulating numbers without thinking deeply about the problem. However, in counting problems, it seems as though searching for patterns through listing in the context of smaller problems can potentially be beneficial. Such activity can help to make an overwhelming problem more attainable.
} 
when we revisited the problem, I gave her the correct Expression GC. Mia was thus in a position of comparing two different expressions to determine which was correct. Mia had some initial intuition about the role of four factorial, but she decided to attempt a smaller case in order to be sure. She worked through a smaller case of dividing six people into two groups of three. She first wrote down A, B, C, D, E, F to represent the people, and she wrote two circles with three dashes each in them. She noted that if she applied her initial method of Expression GI to the smaller case, she would get $\left(\begin{array}{l}6 \\ 3\end{array}\right) \cdot\left(\begin{array}{l}3 \\ 3\end{array}\right)=20$, and she stated that 20, "would not be too bad to write out." This was an important observation; she computed the number of possibilities based on one expression, and she used that activity to begin to write out specific outcomes, which ultimately allowed her to detect how overcounting had occurred. Had this computation yielded a larger result that could not so easily be listed out, I suspect that she may have looked for another option.

Mia then stated that if she applied the other expression (Expression GC) to the situation, she would get 10 . She proceeded to write some examples out to see if she would get 20 or 10 as her answer for the small case. Here we see an instance in which Mia, in the context of the smaller situation, computed totals according to both possible expressions and compared the two. The smaller example allowed her to begin to write particular examples of outcomes, whereas within the original context this could not have been done feasibly.

Mia then wrote out divisions of six students into groups of two, and she wrote out $\mathrm{ABC} \mathrm{DEF}$, then $\mathrm{CEF} \mathrm{ABD}$, and then $\mathrm{CDF} \mathrm{ABE}$ as possible divisions of the students. She paused and then wrote DEF ABC, and something significant happened: Mia noted that this was the same outcome as something she had already written - that is, ABC DEF was the same as DEF ABC. This activity is noteworthy - I contend that the smaller case (and specifically the smaller numbers) enabled Mia to write out some particular outcomes that she otherwise would not have been able to do (she had not written out such outcomes in her work on the original problem).

M: Um, alright A, B, C and then that forces DEF here. So that's one. ABD CEF. ABE and CDF. Hmm. Let's see, oh right, because the first 3 could have been $\mathrm{DEF}$, and then I would have been forced to put $\overline{\mathrm{ABC}}$ in this group, but that's really the same, so these [referring to ABC DEF and DEF ABC] are really the same. Um, oh. Oh, okay so you divide by 2 because, so how would that happen up here? Maybe you chose $\mathrm{ABCDEF}$ here and then in the next slot they could have been chosen this way, that's no different than that. Okay, yeah, I think that this double counts because if I just choose 3 people, it could have been A, B, and C, and then that forces DE and F in the second group. But, let's say the first three people were DEF, that forces $\mathrm{ABC}$ in the second group, and that's exactly the same, just, it doesn't matter, there's, there's, ABC are in a group and DEF are in a group. So this [Expression GI] double counts this, and I think, then, dividing by 4 makes sense. [Note, she went on to say 4 factorial, not 4].

As we see, Mia explained the overcounting by referring to her initial solution; she identified two outcomes as being "exactly the same," as her language underlined above indicates. At the end of the excerpt she returned to the original 20-person problem and considered whether division by 4 factorial made sense. She was ultimately able to 
understand why division by 4 factorial in the original problem made sense and to identify Expression GC as the correct solution.

Using Small Cases to Tackle One Particular Aspect of a Problem Another way in which students utilized small cases was to shed light on one specific aspect of a problem. As an example, we consider Paige's work on the Passwords problem. Paige was enacting a correct total-minus-bad approach, in which she wanted to subtract passwords with exactly zero, one, or two Es from the total number of 8-letter passwords. She quickly determined the number of passwords with exactly zero and exactly one E. As the final part of her total-minus-bad approach, she needed to figure out how to count the number of 8-letter passwords with exactly two Es. Paige realized that in order to solve this part of the problem, she needed to figure out the number of ways to place two Es among eight spots. She felt confident about how to fill in the remaining letters (in $25^{6}$ ways), and so her success on the problem momentarily hinged on one particular part of the problem - placing the two Es in eight spots. Paige suspected that the number of ways to do so involved a permutation, ${ }_{8} P_{2}$, but she was not sure. (Note, this is not correct. Combinations, and not permutations, should be used to place the Es.) In order to check whether this permutation formula was correct (thus allowing her to solve the problem), Paige used a smaller case, which she describes in the underlined section below.

\footnotetext{
P: Wait, is that right? ${ }_{8} P_{2}$. Yeah 'cause order matters, I have, I think that's right. You know what I'm not $100 \%$ sure if this is the right formula, so ... whenever I am unsure of something in a counting problem and I start second guessing myself on like whether or not I'm remembering things correctly,

I: Okay.

P: I usually make a much smaller case, and try and see if this formula matches up with a smaller case that I can actually physically count. So, I'm going to make it smaller, um, instead of 26 letters I'm just going to think about, uh, A, B, and C, and I'm going to have three slots I think, yeah let's have three slots, and I want to know the number of ways I can get let's say 2 Cs. I don't know if this is going to pan out, I might need to do a bigger case ... Um, so I can write out this pretty quickly, oh actually, I'm kind of doing it already for $2 \mathrm{As}, \mathrm{hmm}$, yeah I'll just do it for $2 \mathrm{As}$, since I've already started with my As.
}

As we see in the excerpt above, Paige attempted a smaller problem of 3-letter passwords, using the letters $\mathrm{A}, \mathrm{B}$, and $\mathrm{C}$, and containing at least two As. Over the next several minutes, Paige's work was characterized by writing out 3-letter words and looking for patterns. It is noteworthy, though, that Paige made an error here. She counted out the number of words that had exactly two As, of which there are six (Fig. 1 below shows the 6 words and her computation of ${ }_{3} P_{2}$ ). While this is correct, it is presumably not what she meant to count. She should have simply counted the number of ways of placing exactly two As in three spots (of which there are 3). This had an impact on her work, because as she computed the result using the formula she was testing, ${ }_{3} P_{2}$, it also yielded six. This thus (incorrectly) confirmed her permutation formula for a word of length three.

Thinking her permutation formula was correct for the 3-letter case, Paige then proceeded to check for a word of length four, which took considerably more time. This activity involved detailed listing of 4-letter passwords and looking for patterns as she went. In so doing she realized that regardless of where she placed her two As in the 


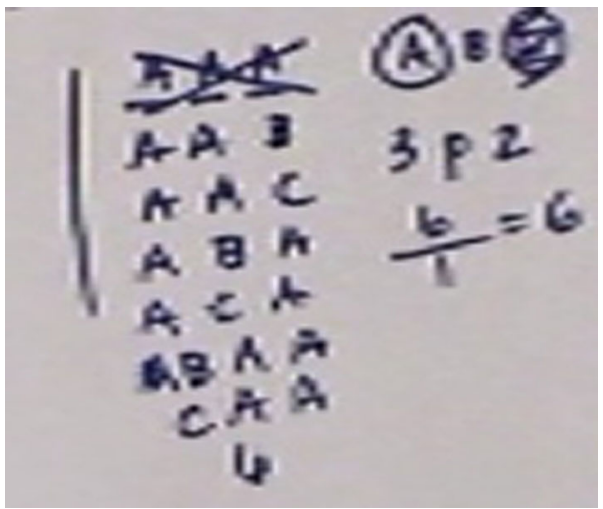

Fig. 1 Paige lists 3-letter words with exactly two As

4-letter password, she always had nine options of how to arrange the remaining letters (which could be Bs or Cs). ${ }^{7}$ We see her language below, and she made a significant realization that actually she wanted to choose spots for the As.

P: Hmm, how can I make that better? So how many different ways could I place, 'cause those were all 9's. So really I just want to know how many different ways I could place 2 As. Oh, well so, if I think of like the slots as like numbered, maybe,

I: Okay.

P: Um, it's like I want to choose the slots, And the order, hmm, does order matter here, I think it might be that I want to, like, know, like I have 4 slots, and I want to know the number of ways I can choose 2 of those slots to put the As in, but then I think I need to divide that answer by 2, because I can't tell the difference between the As.

This was an important step for her, ultimately allowing her to figure out that the number of passwords with exactly two Es was $\left(\begin{array}{l}8 \\ 2\end{array}\right) \cdot 25^{6}$ (and $\operatorname{not}_{3} P_{2} \cdot 25^{6}$ ), and thus to arrive at the correct answer in the original problem.

We see in this example an instance in which a student used a smaller case to tackle a particular part of the problem. Paige's success on the problem was contingent upon the correct solution of one small aspect of the problem (the ways of placing the Es), and she used small cases to figure out the one piece that she really needed. Paige's successful utilization of this strategy involved listing outcomes of a smaller problem, and this allowed her to realize that she wanted combinations, and not permutations, for the number of ways to place Es in the password. This work enabled her to generate the correct answer and ultimately be successful on the Passwords problem. I suggest that Paige's work on the smaller case gave her increased accessibility in dealing with the complicated nature of the problem. Working through particular examples (passwords)

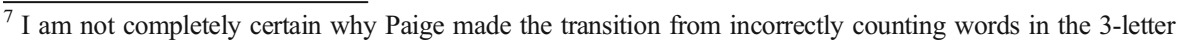
case to correctly counting the ways of placing As in the 4-letter case. I conjecture that because she could not write the entire words as easily as she could in the 3-letter case, she was more inclined to separate out the placing of the As and the filling out of the rest of the word (the Bs and Cs) as two separate processes. Thus, she was less inclined to inadvertently count 4-letter words with two As, but rather she could count the ways of placing two As within a 4-letter word.
} 
enabled her to make progress that she might not otherwise have been able to make with more unwieldy numbers or objects. While I cannot claim that Paige would not have correctly solved the problem without the use of the smaller case, the evidence above indicates that small cases helped her in her work.

\section{Using Smaller Cases to Explain the Difference Between Two Discrepant}

Answers Another way in which students use small cases is to explain the difference between two discrepant answers. This particular use of smaller cases relates very closely to the design of my study (and in fact may be a product of the specific design). As discussed in the Methods section, the study was designed to put students in situations in which they compared two seemingly equivalent answers, both of which seemed to be reasonable (but one of which contained an error).

While the example outlined below is situated particularly within this context, I maintain that it is still a relevant finding, and that it is not merely an isolated incident that could only occur within my particular study. Indeed, I point it out here because students and teachers may often find themselves in situations in which they need to understand or explain why a particular answer is incorrect (imagine a classroom in which two groups of students disagree over two different answers). One of the compelling aspects of counting is that incorrect counting processes can seem logically sound. The example of Anderson's work on the Passwords problem below shows how a careful examination of a smaller case can shed light on key differences between two seemingly-similar counting processes.

As mentioned above, an interesting aspect of the Passwords problem is that Expression PI can seem correct. Here we see that Anderson had also felt Expression PI was correct, and he was trying hard to understand which expression might be wrong. He worked through a smaller case in a very precise and detailed fashion, and this enabled him to perceive an important insight.

In his work on the problem, Anderson found himself in a situation of comparing Expression PC and Expression PI. He took some time to consider it, and as he talked through the expression, he justified to himself why it might make sense; he explained the counting process and said, "based on how it works" he felt that it could make sense.

As Anderson thought more about why the discrepancy occurred, he decided to truncate the situation to examining the number of 4-letter passwords that contain at least three Es, rather than 8-letter passwords that contain at least three Es. Specifically, this came about as he had written down slots and had put $1 \mathrm{~s}$ where an E would go, and $25 \mathrm{~s}$ where a non-E would go. After writing a couple of these slots, he decided to look instead at only four slots instead of dealing with all eight (see Fig. 2). He noted that there were 25 options for any placement of three Es, and he explained again that there were $\left(\begin{array}{l}4 \\ 3\end{array}\right)$ ways to place three Es in the four slots, and then 25 choices for the remaining letter. And then he considered the case in which all four letters were Es, and he stated that there was just one way to do that (this gave him an initial answer of 101).

Then, Anderson engaged in numerical computation for several minutes. He tried the 4-letter problem with the second alternative answer (Expression PI). When he did this, he arrived at $\left(\begin{array}{l}4 \\ 3\end{array}\right) \cdot 26$, which gave an initial answer of 104 . Expression PC had given him an answer of 101, and thus he noted, "the difference is already there". 


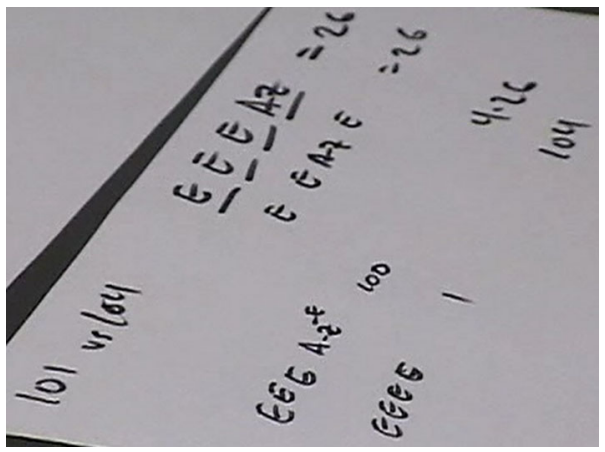

Fig. 2 Anderson writes out a smaller version of the Passwords problem

A: Uh, if I was going to translate to the second one then it becomes, uh, in the four case it becomes $\left(\begin{array}{l}4 \\ 3\end{array}\right)$ equals four, but since the last one doesn't matter, it's 26, which is, uh, blah, 104. Well, let's see. Uh, what was the total on this? It was 100 , and 1 . The difference is already there. It's 101 , versus 104 . Um, okay well since there's already a difference here in the half case, where I'm only taking four letters instead of eight.

This is quite significant. Anderson realized that the discrepancy between the expressions existed even in the smaller problem, and ultimately this enabled him to focus very closely on the small case. This numerical difference of 101 and 104 was small enough for him to be able to consider in detail, and he proceeded to examine the difference here more closely.

The following episode was key for him. First, he said, "I have E, E, E, A through Z. Which is equal to 26". And he wrote down E E E A-Z. Then, he said, "Then I have another E, E, A through Z, E, which is another 26," and he wrote E E A-Z E. Then he said, "And since I do this four times, I have 4 times 26 , which is 104, okay, which would suggest that the second one (Expression PI) is correct" (see Fig. 2 below, the right half of the paper).

Then, nearby (the left half of the paper in Fig. 2), he wrote out E E E A-Z-E, and he said, "I have 3 Es, then I set them to any 25 letters, so let's see, A through Z minus E. And so I have 100 different ways to do that". Then he wrote E E E E and noted "But then I also have 4 Es, and there's only one way to do that." His reflection on this discovery is seen below.

A: Oh, there we go, that's where the difference is. So the difference is, um, yes there's 26 different ways to arrange it so that the first 3 letters are Es, and then the last one can be any of the 26 letters. And then there's another way to arrange it so that the first 2 and the last letter are Es, and the 3rd letter is any letter between A and Z, except if the third letter is an E, it's exactly, it's the exact same case as if the E was the last letter in the first case, which means it's counting multiple passwords twice.

We see that Anderson identified a particular password (the all Es password) that was counted too many times by the incorrect solution. After this, I asked Anderson a couple of questions as a follow up. He gave some sense as to why it took him a while to explain what was wrong with the problem. Simply writing down the range (either $A-Z$ or $A-Z$ excluding E) was not enough for him to see what was happening. His brain was "too lazy to come up with a specific example," and it was not until he came up with that particular example that he could identify the error. This is an insightful self-reflective statement. He was able to identify that a key step in seeing the issue was writing down a specific example 
instead of just writing down a range of choices. I argue that the fact that he was in a smaller case (with fewer letters and slots to consider) facilitated his identification of a particular example.

A: And I was like, oh, the problem - the two methods still come up with different answers, so something must be off on some fundamental level somewhere. Uh, so I realized, well, since my brain's not all that math oriented, I guess I'll just like write it out and see where I go, uh, so let's come up with a few examples, so I was like EEE, and I was like, well, my brain's too lazy to come up with a specific example, so I guess I'll just write down the range, and then I should be okay.... Um, and I guess it's that step that my brain kept skipping due to laziness, (chuckles) that made me overlook that one problem.

After this discussion with the smaller case, Anderson was able to use his work in the small case to make sense of which expression was correct in the original problem. His work through the small case was a vital part of Anderson successfully evaluating the alternative solution and correctly deciding on the correct answer. He used the smaller case to make sense of an apparent discrepancy between two seemingly correct, yet actually different, answers.

Using Small Cases as a Means of Articulation, Communication, and Explanation A final way in which students seemed to use small cases effectively was as a means of communication and explanation. In his work on the Groups of Students problem, Owen provided an example of a way in which a small example served as a means for him to articulate (both to himself and to me) why the incorrect Expression GI had resulted in an overcount. Unlike Mia's or Anderson's work, the small case did not serve strictly as a vital aspect of him figuring out why there was an overcount. As such, he was not using his small case in his execution of the problem, but he used the smaller case as a means to communicate explicitly exactly what had gone wrong.

Owen had initially gotten a solution equivalent to Expression GI (he had left off the last binomial coefficient). When we returned to the problem later in the interview, I gave him Expression GC. He noted that he was "immediately confused" by the 4 factorial, but, having both Expression GI and Expression GC before him, he proceeded to examine which solution might be correct. After examining the solutions for a minute or so, Owen seemed to indicate that he understood what was happening. His language below suggests that he realized overcounting was occurring in some way; in the first underlined section he had figured out what was going on in the problem. However, he chose to describe and explain the answer using eight instead of 20 people.

O: ...Like what if I picked, yeah I see, I see why they did it. What if I picked, okay, oh man I am bad at counting, okay.

I: You're not bad at counting, this is hard.

O: No no, I'm bad. Look, I think this student might be better than me, hold on. If I'm, let me walk through this students' thinking... You pick the five, the four groups... Okay. Um, let me, I don't want to draw 20 dots or 20 things. Let's make it a much smaller situation. Uh, what if it's eight, eight total students.

When he explained it, he made the argument using a smaller case instead of working with the original 20-person problem as stated; that is, he split eight students into four groups of two. Because of his limited language about the actual moment of epiphany, it is difficult to tell if his realization occurred in the 20-person context or the 8-person context. Based on the second underlined phrase above, in which he used the number of 
groups in the original problem (and not the smaller numbers), I suspect that Owen had indeed been thinking in terms of 20 students broken into four groups of five. Also, I do not doubt that Owen's articulation of the smaller case solidified his thinking, and that perhaps talking through the 8-student case helped him to make sense of the situation more than his initial moment of epiphany had allowed (and thus the smaller case served him conceptually and not exclusively as a means of communication). Nonetheless, I argue that this instance highlights the potential ways in which students could use smaller cases as a means of communication and explanation.

It is noteworthy that Owen's choice of a smaller problem numerically reduces parameters but maintains the constraints of the original problem. He wrote down $\mathrm{ABCDEFGH}$, and he wrote the corresponding solution that Expression GI would have yielded with eight students, $\left(\begin{array}{l}8 \\ 2\end{array}\right) \cdot\left(\begin{array}{l}6 \\ 2\end{array}\right) \cdot\left(\begin{array}{l}4 \\ 2\end{array}\right) \cdot\left(\begin{array}{l}2 \\ 2\end{array}\right)$. He then took different colored pens, and, as he explained the solution below, he underlined each binomial coefficient with a different color (pink, red, orange, and green, respectively). The corresponding drawing is seen in Fig. 3.

O: Okay look...I'm going to order my selections, because I think of them as happening in some order. So it's like this first [draws a pink line under $\left(\begin{array}{l}8 \\ 2\end{array}\right)$ ], and I'm going to do this second [draws a red line under $\left(\begin{array}{l}6 \\ 2\end{array}\right)$ ], this third [draws an orange line under $\left(\begin{array}{l}4 \\ 2\end{array}\right)$ ], and this fourth [draws a green line under $\left(\begin{array}{l}2 \\ 2\end{array}\right)$ ], right?

Owen recognized that when he chose the groups of students to be $\mathrm{AB}, \mathrm{CD}, \mathrm{EF}$, and $\mathrm{GH}$, he could have chosen them in some order; the colors represented a particular order in which he chose that division of the 8 people. As he talked about choosing a pair of students, he drew a color under that pair (for example, when he said, "Then I pick C and $\mathrm{D}$ " he drew a red dash under those letters). He drew lines under AB then CD then $\mathrm{EF}$ then $\mathrm{GH}$ in that first example, and he drew $\mathrm{AB}, \mathrm{EF}, \mathrm{GH}, \mathrm{CD}$ in the second.

O: What if, when I first chose 2 students, I picked, I'm going to make it really obvious, A and B [draws a pink line under $\mathrm{AB}$ ]... Cool, then I have 6 remaining. Then I pick $\mathrm{C}$ and $\mathrm{D}$ [draws a red line under $\mathrm{CD}$ ]. Oh, what if I have 4 remaining? Oh I'm going to pick these 2 [draws an orange line under EF, then draws a green line under $\mathrm{GH}]$.

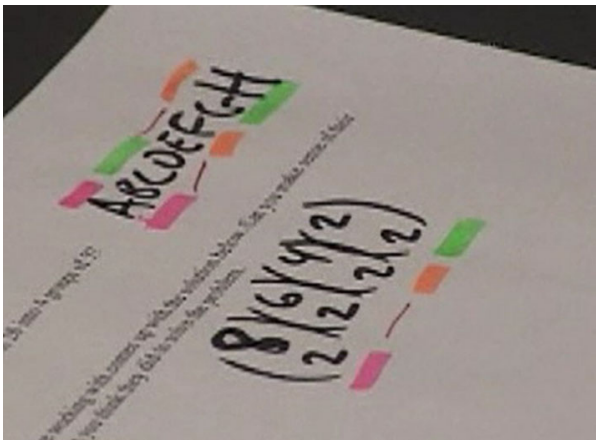

Fig. 3 Owen's color-coded explanation of the reduced Groups of Students problem 
He then noted that he could pick the same division of 8 people $(\mathrm{AB}, \mathrm{CD}, \mathrm{EF}, \mathrm{GH})$ in another way, and he drew colors above the letters to represent picking the pairs in a different order. This time, he drew AB first, then EF, then GH, then $\mathrm{CD}$. When he says, "I could have picked CD or I could have picked EF" he referred to the different colors he drew below and above the letters, respectively.

O: Well, that's one way to count, pick 'em, right? Color, change the colors drawn above again. So you can put the pairs, you can pick the pairs in different orders... To make it obvious I'll draw the colors. Look, I'm just drawing the same exact pairs, different colors up here... To symbolize that if I did this, I could have picked him, this same pair twice, right, but second I could have picked CD or I could have picked EF.

I: Sure.

O: And then third I could have picked EF or GH...And last, CD or GH, the remaining pairs...Same exact pairs, counted twice here, counted only once here because you're dividing out the ordering of the pairs... So this is the order of the pairs, this is my overcounting, overcounting here, this is the number of ways to order 4 things, this way you get rid of those double counts.

Owen's smaller case had a communicative and explanatory element to it. That is, it seems as though the smaller case facilitated his articulation of what exactly went wrong. It is not to say that Owen could not also have easily explained his work (and the overcount) using the original problem, but he chose not to do so. And the smaller case, which was easier to write out, was certainly effective in helping him to explain key aspects of the problem and the incorrect solution. The smaller case thus served as a useful form of communication for Owen.

As another example of this phenomenon, Mike had solved the Groups of Students problem without using a smaller case. In discussing his work, Mike indicated that if asked to explain the solution to someone else, he would talk through a smaller example, noting, "I would just say that you could make a smaller example with just, we'd want to do at least 3 groups to show why it has to be a factorial, I suppose, and so you'd have 3 groups of maybe 6 objects." So while solving a smaller problem was not a part of his solution process, he seemed to recognize that using a smaller problem could be an effective means of explanation. This kind of communication seems to have a natural relationship with teaching. Like Mike, a number of students commented that if they had to explain their work to students in a classroom setting, they would consider small cases. This seemed to be because these students saw small cases as allowing for actually listing out some outcomes, which could help students get a sense of the problem. Pedagogical ideas are discussed in the section on the avenues for further research.

\section{Potential Pitfalls in Working with Small Cases}

The results above highlight various ways in which students used the problem solving strategy of smaller cases, and these results emphasize the potential benefit of such a strategy for solving counting problems. However, there were also instances in the data that suggested the strategy could lead to negative consequences if it was not implemented properly. Below, I draw attention to three such pitfalls that arose during the study. These are meant to point out what can happen if the strategy is not carried out with care and precision. 
Mathematical Properties Between the Original Problem and the Smaller Problem

May Differ It can be difficult to reduce a problem to a smaller problem that maintains the same mathematical features of the original, and combinatorial problems can be particularly susceptible to such a challenge. As discussed previously, the numbers 100 and 3 have different properties; reducing a problem dealing with 100 objects to a problem dealing with 3 objects could potentially change some subtle mathematical features of the problem. As students numerically reduce some parameter in order to create a smaller problem, some typically unforeseen mathematical property can arise (or disappear) that had been absent (or present) in the original problem.

As a specific example, this issue arose in Paige's work, when she evaluated the correct alternative answer to the Groups of Students problem. Expression GC has a 4 factorial in the denominator, while Expression GI does not, and when Paige compared the two answers, she sought to examine whether division by 4 factorial was necessary. On this problem Paige had reduced the problem to splitting into two groups; had she applied Expression GC to such a case, it would have meant dividing by 2 factorial. However, two and two factorial are equal, and simply by looking at one small case she could not clearly tell whether she was dividing by two or by two factorial (and thus whether she should divide by four or 4 factorial in the original problem). While the reduction of the problem from dealing with four groups to two groups undeniably made the outcomes easier to list, a specific property about the number 2 (that does not apply to the number 4) affected Paige's ability to generalize from the smaller case. Fortunately, Paige was able to recognize 2's special property, and she sought other means of reasoning through why 4 factorial made sense.

This example highlights the fact that different numbers have properties that may cause them to behave in certain ways, and reducing parameters to make a smaller problem can enhance some properties or inhibit others. It takes mathematical knowledge and experience to foresee how certain numerical properties could affect the constraints of the problem, and this is issue is something of which students ought to be aware.

\section{The Relationship Between the Smaller Problem and the Original Problem Can be}

Lost Another potential danger is that students should not become so involved in a smaller case that they are unable to relate it back to the original problem. For example, in her work on the Passwords problem, Jenny spent a considerable amount of time working through a small example to make sense of a particular part of the problem. At one point, she was trying to remind herself of the ways of placing the remaining non-E letters, and to address this issue she looked at a case of only 3 letters. In the course of this work, she drew a very detailed tree diagram and spent a considerable amount of time working through that diagram. Eventually, this work had the effect of distracting her from what she had initially been doing. She had difficulty connecting her work on the small case back to the original problem, and to recall how those endeavors particularly related to what she was trying to accomplish. This is not to say that detailed and lengthy forays into smaller cases are always bad - Anderson, for example, showed instances of very effective work with small cases that could be called tangential. However, such work is only effective insofar as it can be directly related back to the problem at hand. 
The Smaller Problem Can be Worked Carelessly, Without Precision and Attention to Detail A final point is that effectively working through small cases often requires students to be precise and methodical. This is not new advice in the problem solving literature; Polya (1945) emphasizes this in his famous book, How to Solve It (e.g., pp. 68-69). This is particularly noteworthy in counting problems, though, when numbers can be large and maintaining the integrity of the original problem can be difficult. In the examples I have described of successful implementation of small cases, these students' work was marked by very precise, detailed work. The students did not seem in a hurry just to apply a formula, get an answer, and be done. Rather, they invested time (often more than $15 \mathrm{~min}$ ) in figuring out the problem, and they were willing to try a couple of cases and to engage in systematic listing. While these are certainly desirable qualities for mathematics students in a variety of settings and domains, these are particularly important attributes when dealing with complex counting problems.

\section{Conclusions and Avenues for Further Research}

\section{Conclusions}

In drawing conclusions from the results above, I first point out that the strategy of using small cases in the context of combinatorial tasks is related to sets of outcomes. In prior work, I indicated the importance of considering sets of outcomes for students as they count, suggesting that much benefit could be afforded by explicitly utilizing sets of outcomes in the activity of counting (Lockwood 2013, 2014; see also Hadar and Hadass 1981; Polaki 2005). The findings in this paper build upon this notion, indicating that students' uses of smaller cases, in particular their use of systematic listing, was related to their facility with the set of outcomes. In some instances, the use of a smaller problem allowed for work with the set of outcomes that might not otherwise have been attainable.

Second, I contend that the utilization of smaller cases is particularly useful in the domain of combinatorial enumeration. The strategy of using smaller cases can be a powerful problem-solving resource, and the nature of counting problems makes them particularly appropriate for the strategy of using smaller, similar problems. Specifically, in counting problems, sets of outcomes are often so large that they can be difficult to conceive of and manage (for example, the answer to the Passwords problem is over 575 million, and the answer to the Groups of Students problem is approximately 488 million). Smaller cases can reduce the magnitude of such sets and can make the problems and the solution sets more accessible. Additionally, it is very important for a student to be able to articulate what he or she is trying to count, and smaller cases can particularly facilitate such activity. In some counting problems the objects themselves that are being counted can be quite difficult to articulate (for example, in the Groups of Students problem, an outcome is one partition of 20 students into four groups of five; this necessitates coordinating a number of factors -20 distinct students, what such a division might look like, and how they might be divided to create a desirable division). Considering these difficulties with manipulating large sets of outcomes and articulating 
those outcomes, smaller cases are particularly useful because they allow not only for the magnitude of the outcomes to be reduced, but often also for the outcomes themselves to be easier to identify. Counting problems are complex problems that are easy to state but can be difficult to solve, and they tend to involve answers of great magnitude that cannot easily be checked. As such, the use of small cases provides a way to ground a student's work meaningfully.

Finally, given the complexity of counting problems, another finding is that the strategy of using small cases can be useful but requires care. When a smaller problem is utilized, students must be aware of any subtleties or complexities of the original problem. They must attend both to the parameters and the constraints of the problem, being aware of potentially significant mathematical properties that might affect their work. Students must also be precise and methodical in their work. The general positive qualities of a careful mathematics student become especially valuable in the context of using smaller problems on combinatorial tasks.

\section{Avenues for Further Research}

The results discussed above highlight ways in which students used smaller cases in their work on combinatorial problems. Overwhelmingly, the strategy helped the students who chose to implement it, in spite of the potential drawbacks they could have encountered. Given student difficulties with counting problems, the use of smaller cases seems to be a promising domain-specific strategy that could be useful for students. However, beyond the results presented above, at this point there is not much known about students' uses of this strategy, particularly in the combinatorial context. I discuss potential avenues for further research that could stem from this initial work.

One potential direction for further study is to conduct research that clearly targets how students think about and reflect upon their uses of small cases. Little is currently known about whether students recognize the potential value of such a strategy, whether they see the potential downsides, and whether it is a strategy that they employ across mathematical domains. Such reflection about the strategy could give insight into students' current thinking and beliefs about such strategies, particularly in the context of combinatorial problems. Studies could be conducted that specifically target students' thinking about their uses of smaller cases, perhaps presenting interventions in which students are explicitly instructed to reflect upon their use of small cases in their problem solving work.

Another possible avenue of further study is to investigate effective ways to develop this strategy among students. This is not a trivial endeavor - successful use of the strategy can be overwhelming, because of some of the potential disadvantages mentioned above. Schoenfeld (1979) presented evidence that the problem solving heuristic of solving a similar problem was in fact one that could effectively be taught. That is, some of his students who received explicit instructed about the strategy went on to utilize it successfully. There is thus precedence for explicit instruction of the strategy, and a study could be conducted about the effectiveness of teaching the strategy in the context of combinatorics. Such research could begin with teaching experiments and could extend into curricula development and classroom implementation. Related to this, students' uses of small cases seem to be related to the verification of combinatorial problems, as Eizenberg and Zaslavsky (2004) suggest. Due to large sets of outcomes, 
combinatorial problems are notoriously difficult to verify; however, the use of smaller cases could be presented as a verification strategy for students.

Also, it could be worthwhile to study pre-service teachers' knowledge and beliefs related to this strategy. This is relevant both for understanding their own notions of such a strategy, but also to gain insight into how they might implement the strategy in the classroom. Researchers could learn more about how teachers could foster the use of such a strategy among students. I would advocate such an investigation within the specific mathematical domain of combinatorics, but it may be worthwhile to look at the strategy more generally as well.

\section{References}

Auerbach, C., \& Silverstein, L. B. (2003). Qualitative data: An introduction to coding and analysis. New York: New York University Press.

Batanero, C., Navarro-Pelayo, V., \& Godino, J. (1997). Effect of the implicit combinatorial model on combinatorial reasoning in secondary school pupils. Educational Studies in Mathematics, 32, 181-199.

Eizenberg, M. M., \& Zaslavsky, O. (2004). Students' verification strategies for combinatorial problems. Mathematical Thinking and Learning, 6(1), 15-36.

Fontana, A., \& Frey, J. H. (2005). Dealing with compound events. In N. K. Denzin \& Y. S. Lincoln (Eds.), The sage handbook of qualitative research (3rd ed., pp. 695-727). Thousand Oaks: Sage Publications, Inc.

Godino, J., Batanero, C., \& Roa, R. (2005). An onto-semiotic analysis of combinatorial problems and the solving processes by university students. Educational Studies in Mathematics, 60, 3-36.

Hadar, N., \& Hadass, R. (1981). The road to solve combinatorial problems is strewn with pitfalls. Educational Studies in Mathematics, 12, 435-443.

Jones, G. (Ed.). (2005). Exploring probability in school: Challenges for teaching and learning. New York: Springer.

Kavousian, S. (2008). Enquiries into undergraduate students' understanding of combinatorial structures. Unpublished doctoral dissertation. Vancouver: Simon Fraser University.

Lobato, J. (2003). How design experiments can inform a rethinking of transfer and vice versa. Educational Researcher, 32(1), 17-20.

Lobato, J. (2012). The actor-oriented transfer perspective and its contributions to educational research and practice. Educational Psychologist, 47(3), 232-247.

Lockwood, E. (2011a). Student approaches to combinatorial enumeration: The role of set-oriented thinking. (Unpublished doctoral dissertation). Portland: Portland State University.

Lockwood, E. (2011b). Student connections among counting problems: an exploration using actor-oriented transfer. Educational Studies in Mathematics, 78(3), 307-322. doi:10.1007/s10649-011-9320-7.

Lockwood, E. (2013). A model of students' combinatorial thinking. The Journal of Mathematical Behavior, 32, 251-265. doi:10.1016/j.jmathb.2013.02.008.

Lockwood, E. (2014). A set-oriented perspective on solving counting problems. For the Learning of Mathematics, 34(2), 31-37.

Lockwood, E., \& Caughman, J. S. (2015). Set partitions and the multiplication principle. Accepted with revision, Problems, Resources, and Issues in Mathematics Undergraduate Studies.

Lockwood, E., \& Gibson, B. (2014). The value of systematic listing in correctly solving counting problems. In T. Fukawa-Connelly, G. Karakok, K. Keene, \& M. Zandieh (Eds.), Proceedings for the Seventeenth Special Interest Group of the MAA on Research on Undergraduate Mathematics Education (pp. 153168). Denver: Northern Colorado University.

Maher, C. A., \& Martino, A. (1996). Young children invent methods of proof: The gang of four. In P. Nesher, L. Steffe, P. Cobb, B. Greer, \& J. Goldin (Eds.), Theories of mathematical learning (pp. 431-447). Manwah: Lawrence Erlbaum Associates.

Maher, C. A., \& Speiser, R. (2002). How far can you go with block towers? The Journal of Mathematical Behavior, 16(2), 125-132.

Maher, C. A., Powell, A. B., \& Uptegrove, E. B. (Eds.). (2011). Combinatorics and reasoning: Representing, justifying, and building isomorphisms. New York: Springer.

Martin, G. E. (2001). The art of enumerative combinatorics. New York: Springer. 
National Council of Teachers of Mathematics. (1989). Curriculum and evaluation standards for school mathematics. Reston: NCTM.

National Council of Teachers of Mathematics. (2000). Principles and standards for school mathematics. Reston: NCTM.

National Governors Association Center for Best Practices \& Council of Chief State School Officers. (2010). Common core state standards. Washington, DC: Authors.

Polaki, M. V. (2005). Dealing with compound events. In G. A. Jones (Ed.), Exploring probability in school: Challenges for teaching and learning (pp. 191-214). New York: Springer.

Polya, G. (1945). How to solve it. Princeton: Princeton University Press.

Roa, R. (2000). Razonamiento combinatorio en estudiantes con preparacion matematica avanzada. (Unpublished Doctoral dissertation). Universidad de Granada.

Schoenfeld, A. (1979). Explicit heuristic training as a variable in problem-solving performance. Journal for Research in Mathematics Education, 10(3), 173-187.

Schoenfeld, A. (1980). Teaching problem-solving skills. American Mathematical Monthly, 87, 794-805.

Silver, E. (1979). Student perceptions of relatedness among mathematical verbal problems. Journal for Research in Mathematics Education, 10(3), 195-210.

Silver, E. (1981). Recall of mathematical problem information: solving related problems. Journal for Research in Mathematics Education, 12(1), 54-64.

Sriraman, B., \& English, L. D. (2004). Combinatorial mathematics: research into practice. Mathematics Teacher, 98(3), 182-191.

Strauss, A., \& Corbin, J. (1998). Basics of qualitative research: Techniques and procedures for developing grounded theory (2nd ed.). Thousand Oaks: Sage Publications, Inc.

Tucker, A. (2002). Applied combinatorics (4th ed.). New York: Wiley.

Willis, G. B. (2005). Cognitive interviewing: A tool for improving questionnaire design. Thousand Oaks: Sage Publications, Inc. 